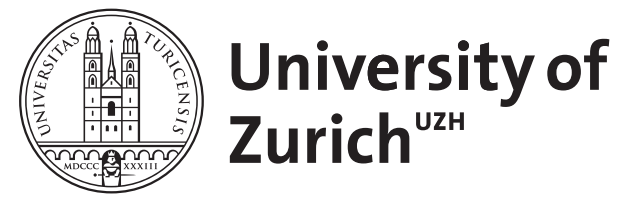
Archive

University of Zurich

University Library

Strickhofstrasse 39

CH-8057 Zurich

www.zora.uzh.ch

Year: 2015

The biogeography of adaptive radiations and the geographic overlap of sister species

Pontarp, Mikael ; Ripa, Jörgen ; Lundberg, Per

DOI: https://doi.org/10.1086/683260

Posted at the Zurich Open Repository and Archive, University of Zurich

ZORA URL: https://doi.org/10.5167/uzh-118347

Journal Article

Published Version

Originally published at:

Pontarp, Mikael; Ripa, Jörgen; Lundberg, Per (2015). The biogeography of adaptive radiations and the geographic overlap of sister species. The American Naturalist, 186(5):565-581.

DOI: https://doi.org/10.1086/683260 


\section{CHICAGO JOURNALS}

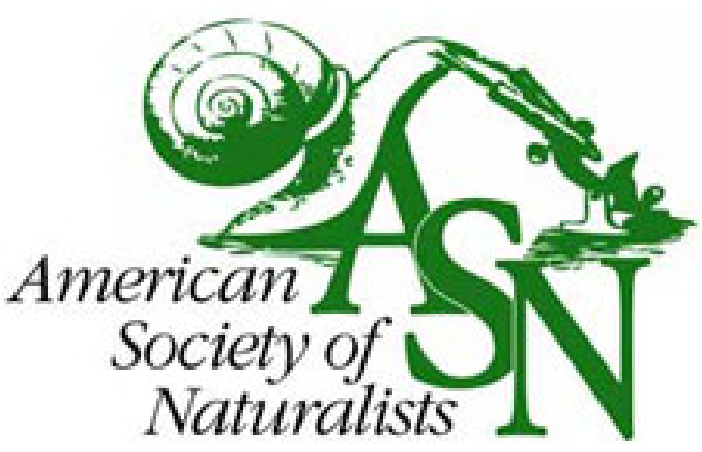

The University of Chicago

The Biogeography of Adaptive Radiations and the Geographic Overlap of Sister Species Author(s): Mikael Pontarp, Jörgen Ripa and Per Lundberg,

Source: The American Naturalist, (-Not available-), p. 000

Published by: The University of Chicago Press for The American Society of Naturalists

Stable URL: http://www.jstor.org/stable/10.1086/683260

Accessed: 28/09/2015 13:00

Your use of the JSTOR archive indicates your acceptance of the Terms \& Conditions of Use, available at http://www.jstor.org/page/info/about/policies/terms.jsp

JSTOR is a not-for-profit service that helps scholars, researchers, and students discover, use, and build upon a wide range of content in a trusted digital archive. We use information technology and tools to increase productivity and facilitate new forms of scholarship. For more information about JSTOR, please contact support@jstor.org. 


\title{
The Biogeography of Adaptive Radiations and the Geographic Overlap of Sister Species
}

\author{
Mikael Pontarp, ${ }^{1,2, \star}$ Jörgen Ripa, ${ }^{2}$ and Per Lundberg ${ }^{2}$ \\ 1. Institute of Evolutionary Biology and Environmental Studies, University of Zurich, Winterthurerstrasse 190, 8057 Zurich, Switzerland; \\ 2. Department of Biology (Theoretical Population Ecology and Evolution Group), Ecology Building, Lund University, SE-223 62 Lund, \\ Sweden \\ Submitted November 14, 2014; Accepted June 22, 2015; Electronically published September 16, 2015 \\ Online enhancement: appendix.
}

\begin{abstract}
AвSTRACT: The biogeography of speciation and what can be learned about the past mode of speciation from current biogeography of sister species are recurrent problems in evolution. We used a trait- and individual-based, eco-evolutionary model to simulate adaptive radiations and recorded the geographical overlap of species during and after evolutionary branching (speciation). We compared the spatial overlap among sister species in the fully saturated community with the overlap at the speciation event. The mean geographic overlap at speciation varied continuously from complete (sympatry) to none (allopatry), depending on local and regional environmental heterogeneity and the rate of dispersal. The distribution of overlap was, however, in some cases considerably bimodal. This tendency was most expressed at large values of regional heterogeneity, corresponding to sharp environmental contrasts. The mean geographic overlap also varied during the course of a radiation, sometimes with a consistent negative trend over time. The speciations that resulted in currently observable end community sister species were therefore not an unbiased sample of all speciations throughout the radiation. Postspeciation range shifts (causing increased overlap) occurred most frequently when dispersal was high or when local habitat heterogeneity was low. Our results help us understand how the patterns of geographic mode of speciation emerge. We also show the difficulty in inferring the geographical speciation mode from phylogenies and the biogeography of extant species.
\end{abstract}

Keywords: ecological interactions, evolutionary dynamics, speciation, adaptive radiation, metacommunity, individual-based modeling.

\section{Introduction}

Speciation can be characterized by two critical steps: the evolution of ecological divergence, which ensures longterm coexistence, and reproductive isolation, which keeps the gene pools separate and the species proper (Coyne and Orr 2004). The relative importance of the two processes and the biotic and abiotic drivers of speciation has been

\footnotetext{
* Corresponding author; e-mail: mikael.pontarp@biol.lu.se.
}

Am. Nat. 2015. Vol. 186, pp. 000-000. (C) 2015 by The University of Chicago. 0003-0147/2015/18605-55894\$15.00. All rights reserved.

DOI: $10.1086 / 683260$ studied and debated for more than a century. Because long (evolutionary) timescale processes are inherently difficult to study, indirect methods to infer the dominating processes of speciation have been used, although often on the basis of questionable assumptions (e.g., Losos and Glor 2003).

It has long been argued that reproductive isolation most easily evolves if the incipient species are geographically isolated, to allow for the necessary genetic linkage between ecological and mating traits (Mayr 1942; Felsenstein 1981a). It can also be argued that the spatial distribution of newly formed types, on the way to speciation, plays a large role for the ecological forces driving the necessary ecological divergence. Several theoretical studies support these ideas by showing that a spatially heterogeneous environment with limited dispersal may generate the necessary disruptive selection for the evolution of habitat specialists (Brown and Pavlovic 1992; Day 2000; Parvinen and Egas 2004; Nilsson and Ripa 2010). In addition, empirical studies show that island size (Losos and Schluter 2000) and local habitat heterogeneity (Hobohm 2000; Roos et al. 2004; Hughes and Eastwood 2006) are positively related to local speciation rate. In contrast, several natural systems with low habitat heterogeneity, few geographical barriers, and high dispersal (e.g., marine systems) can contain a vast diversity (Rocha et al. 2005; Krug 2011), implying that ecological mechanisms such as resource specialization and coevolution are in some cases the main drivers of speciation. In fact, spatial separation may be the direct consequence of habitat specialization, either as a result of coupled evolution of habitat preferences (Fry 2003; Gavrilets and Vose 2005) or philopatry (Kisdi 2002) or because dispersing individuals fail to establish in competition with locally adapted types (DeMeester 1996; Ogden and Thorpe 2002; Dieckmann et al. 2004; Nosil et al. 2009). The spatial separation of different lineages may thus be both a cause and a consequence of the ecological divergence.

The geographic distribution of closely related species is often used to infer underlying evolutionary mechanisms, 
despite its ambiguities. For example, species ranges are used for ecological niche modeling (Elith and Leathwick 2009) to study patterns of niche conservatism, that is, whether closely related species are also close in niche space (e.g., Peterson et al. 1999; Eaton et al. 2008; Nakazato et al. 2010; Couvreur et al. 2011). Also, the geographical overlap of sister species, especially as a function of time since speciation, has been used to assess the biogeography of the speciation process (Barraclough and Vogler 2000; Nakazato et al. 2010; Couvreur et al. 2011; Quenouille et al. 2011). This approach has been criticized because species ranges may shift several times on the typical timescale of phylogenies, producing a noisy pattern with little information about the biogeography at the time of speciation (Losos and Glor 2003; Fitzpatrick and Turelli 2006). On this note, studies have used simulations to investigate how different types of range changes may affect the link between end community patterns and the geographical overlap among newly formed species (e.g., Barraclough and Vogler 2000; Phillimore et al. 2008). It can also be questioned to what extent extant sister species represent an unbiased sample of all speciation events from a particular phylogeny. During an adaptive radiation (the rapid diversification of a single ancestral species, driven by ecological opportunity, within a bounded geographic region), it can be expected that competition with congeners plays an increasingly important role as the biodiversity builds up. Consequently, speciation events that occur late in a radiation, typically resulting in extant sister species, take place under different ecological conditions compared with early speciations and thus may not be representative for the whole clade (Quenouille et al. 2011).

We used a trait- and individual-based eco-evolutionary model, introduced by Day (2000) and extended by Pontarp et al. (2012b), with a spatially explicit environment to investigate the biogeography of speciation events during an adaptive radiation. We defined species as clusters of individuals having similar traits, and we viewed a cluster that branched into two distinct clusters in trait space as a speciation event. With this as background, we simulated adaptive radiations of competitive metacommunities - a set of local communities connected by dispersal (Leibold et al. 2004; Urban and Skelly 2006; Urban et al. 2008). First, we asked how dispersal, the number of available niches within a habitat (here referred to as local heterogeneity), and differences between habitats (here referred to as regional heterogeneity) affect the geographical overlap during speciation throughout the adaptive radiations. Second, we investigated how well the spatial overlap of sister species in the saturated and fully evolved metacommunities corresponds to the overlap at speciation of those species and whether the biogeography of those sister species is representative of speciations across the entire adaptive radiation.
Finally, we studied postspeciation range shifts and whether classical measures of the geographical overlap of end community sister species as a function of time since speciation can be used for inference of the biogeographic mode of past speciation events (Barraclough and Vogler 2000; Nakazato et al. 2010; Couvreur et al. 2011; Quenouille et al. 2011).

\section{Methods}

Similar to several earlier models of competitive interactions and the evolution of resource specialization (Christiansen and Loeschcke 1980; Brown and Vincent 1987; Dieckmann and Doebeli 1999; Haller et al. 2013), we assume that local fitness of a particular phenotype is a function of a single, continuous, evolving trait (e.g., body size) that maps onto a unimodal resource distribution. We use the distribution of trait values to define species and speciation events: clusters of phenotypically similar individuals are viewed as species, and clusters branching into two distinct clusters are registered as speciation events (see details below).

The spatial structure in our model consists of five distinct habitats connected by stepping stone dispersal, each with a unique resource distribution. Individuals reproduce according to their local fitness, and a proportion of the individual offspring disperse to adjacent habitats. Taken together, the habitats constitute a regional environment sufficiently coarse grained to allow for the buildup of a reasonably speciose metacommunity (Leibold and Norberg 2004; Urban 2004; Thompson 2005). If the overlap of the resource distributions among habitats is large, they are ecologically similar, and the entire region has relatively low regional heterogeneity. Conversely, if the overlap of the resource distributions is low, the regional heterogeneity is high (fig. 1).

Individuals reproduce according to their fitness, adapt to their biotic and abiotic environment through mutations, and disperse in space. The adaptive radiation and the geographical distribution of individuals are driven by microevolution, and speciation and extinctions are thus emergent properties of this process.

\section{The Model}

The use of local resources for survival and reproduction is defined by the organism's ecological trait value $(z)$. This trait maps onto the local resource distribution and determines which range of the resources that an individual can efficiently utilize. Keeping the size analogy, the trait $(z)$ can, for example, be interpreted as the beak size of a granivorous bird that utilizes some part of a size distribution of seeds on an island. Individuals that have similar traits 

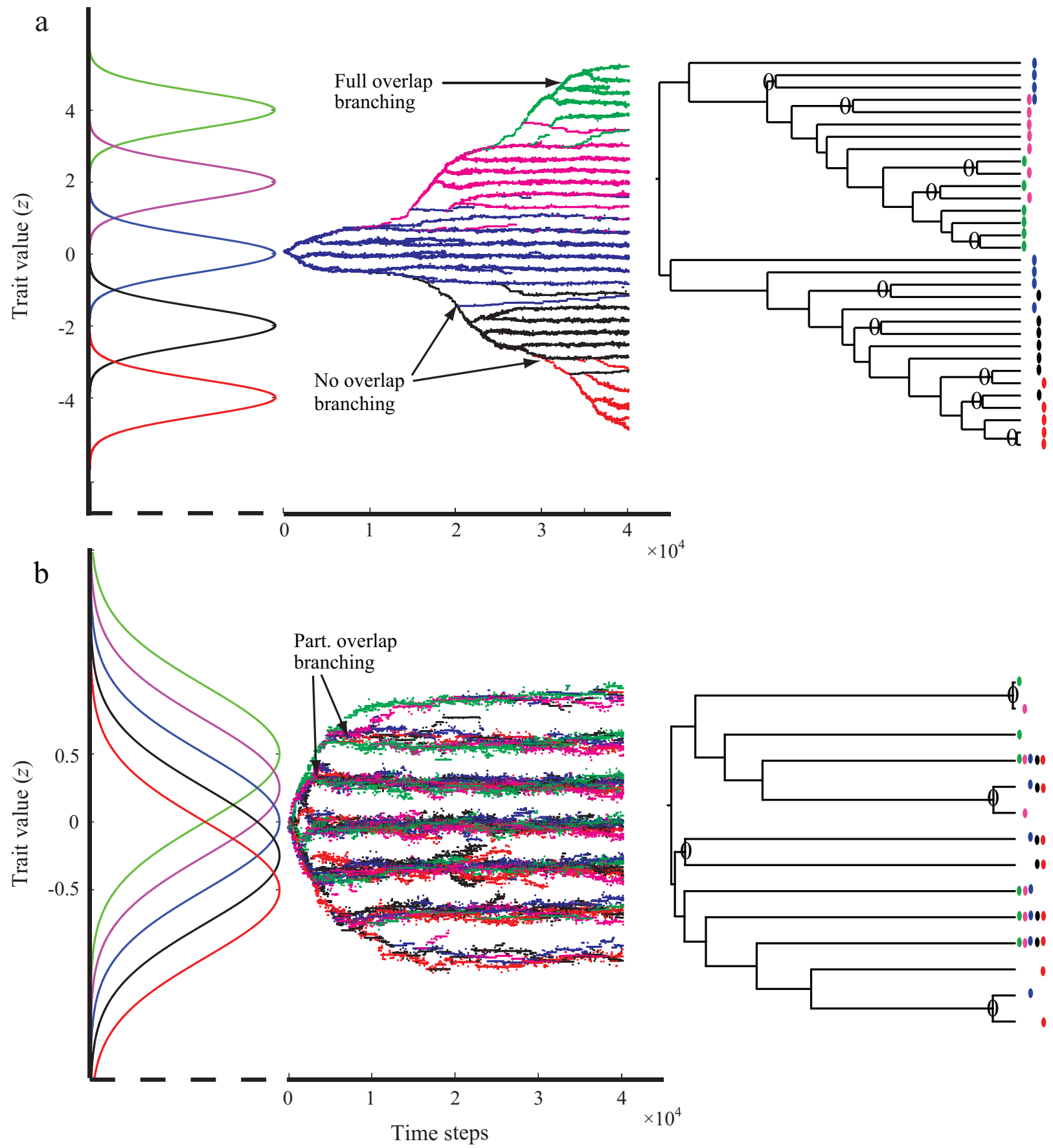

Figure 1: Examples of simulated adaptive radiations with the corresponding reconstructions of the phylogenetic trees for high $(a)$ and low (b) regional heterogeneity. Each colored dot in the figures corresponds to an individual in time ( $Y$-axis) and trait space $(X$-axis). The color indicates in which habitat the individual occurs. Nodes and branch lengths in the tree are derived from branching events and time steps between them. Color coding of leaves and circles in the phylogeny denote habitat occupancy and nodes that correspond to sister species, respectively. The resource distributions for each color-coded habitat mapped on the trait axis are shown at the left. Modified from Pontarp et al. (2012b) and chosen for illustration; other parameters can give less distinct clusters. 
in our model compete more for shared resources than less similar ones.

Local fitness (the expected number of offspring) of a focal individual is a function of its trait value (matching the local resource distribution), the traits of all other individuals in the same habitat (utilizing the same resources), and the local resource distribution. These assumptions are expressed in a model of local fitness according to a fitness generating function ( $G$ function) derived from the classical Lotka-Volterra competition model (Vincent and Brown 2005):

$$
G\left(z, \mathbf{z}, Z_{i}\right)=1+r\left[1-\frac{\sum_{j} \alpha\left(z, z_{j}\right)}{K\left(z, Z_{i}\right)}\right],
$$

where

$$
\begin{aligned}
& K\left(\mathrm{z}, Z_{i}\right)=K_{0} e^{-\left[\left(\mathrm{z}-Z_{i}\right)^{2} / 2 \sigma_{K}^{2}\right]}, \\
& \alpha\left(z, z_{j}\right)=e^{-\left[\left(z-z_{j}\right)^{2} / 2 \sigma_{\alpha}^{2}\right]},
\end{aligned}
$$

where $z$ is the trait value of the focal individual, $\mathbf{z}$ is a vector of trait values of all individuals in the same habitat (including the focal individual), and $Z_{i}$ is the local optimal trait value in habitat $i$. The parameter $r$ denotes the intrinsic growth rate (equal for all individuals), and $K\left(\mathrm{z}, Z_{i}\right)$ is the carrying capacity as experienced by the focal individual. $K_{0}$ denotes the maximal carrying capacity (at $z=Z_{i}$ ), and the resource availability falls off symmetrically as $z$ deviates from $Z_{i}$ according to the width of the resource distribution $\left(\sigma_{K}\right)$. The function $\alpha\left(z, z_{j}\right)$ gives the competition coefficient between the focal individual and a competitor with trait $z_{j}$, standardized such that $\alpha\left(z_{j}, z_{j}\right)=1$ and $0<\alpha\left(z_{i}, z_{j}\right)<1$ $\left(z_{i} \neq z_{j}\right)$. If all individuals have the same $z$ value, the sum in equation (1) evaluates to the number of individuals in the same habitat, and equation (1) gives the per capita growth rate of the discrete time logistic equation. Finally, competition declines with distance in trait space according to a Gaussian function with standard deviation $\sigma_{\alpha}$ (eq. [3]).

Each habitat's resources are distributed and centered around a unique position, $Z_{i}$, on the trait axis, positioned such that the habitats are lined up with a fixed interval, $\Delta Z$, between resource peaks (fig. 1). Neighboring habitats in trait space are also considered neighbors in real space. Between reproductive events, each individual disperses with a probability $d$ to a randomly chosen neighboring habitat (stepping-stone dispersal). The dispersal rate $d$ can be interpreted as a measure of geographical distance between habitats or the dispersal propensity of the organism.

The relative magnitudes of the parameters defined above determine local and regional heterogeneity (Pontarp et al. $2012 b)$. The width of the resource distribution $\left(\sigma_{K}\right)$ and the distance between resource peaks $(\Delta Z)$ determine the degree of resource overlap between habitats in trait space (fig. 1).
If the distance between resource peaks is large and the width of the distributions is small, then the habitats are ecologically distinct with small resource overlap. The quotient $\Delta Z / \sigma_{K}$ is thus a measure of habitat differences in terms of resource type-here referred to as regional heterogeneity.

A large $\sigma_{\alpha}$ leads to relatively high degree of competition between individuals with similar traits compared with a small $\sigma_{\alpha}$ (Geritz et al. 1998). In this sense, $\sigma_{\alpha}$ represents niche width, and the quotient $\sigma_{K} / \sigma_{\alpha}$ can be interpreted as the potential for local niche differentiation among individuals or species - here referred to as local heterogeneity. As described by Pontarp et al. (2012b), $\sigma_{K} / \sigma_{\alpha}$ affects the branching process. If $\sigma_{\alpha}$ is larger than or close to $\sigma_{K}$, there will only be room (in terms of niche space) for one species (phenotypic cluster) per habitat and no branching will occur in the local community. On the other hand, if $\sigma_{\alpha} \ll \sigma_{K}$, local evolutionary branchings are facilitated (Geritz et al. 1998; Dieckmann and Doebeli 1999).

\section{Simulations}

Following Pontarp et al. (2012b), we used the model described above to simulate adaptive radiations in spatially distributed habitats, using different settings of regional heterogeneity (range 0.01-0.5), local heterogeneity (range 1$10)$, dispersal rate $\left(d=10^{-5}\right.$ or $\left.d=10^{-3}\right)$, and mutation rate $\left(\mu=10^{-4}\right.$ or $\left.\mu=10^{-3}\right)$. Other settings, unless otherwise stated, were $r=1, K_{0}=1,000, \sigma_{K}=1$. An individual that is optimally adapted to its environment gets, according to these parameters, two offspring per time step when not affected by competition, and its progeny will grow to a local population size of 1,000 , disregarding dispersal and competition from other morphs. Simulations were run in MATLAB version 7.12.0 at the Swedish National Infrastructure for Computing at the Center for Scientific and Technical Computing at Lund University.

In the beginning of each simulation, the middle habitat was seeded with 10 optimally adapted individuals (different initial conditions were also tested; see "Initial Conditions" in the appendix; appendix available online). Simulations proceeded in alternating phases of reproduction and dispersal each generation (time step). In the reproduction phase, each individual produced a Poisson-distributed number of offspring with a mean equal to the individual's fitness (eqq. [1]-[3]). Each offspring inherited the $z$ value of its parent, unless they mutated with a probability $\mu$ to a slightly different trait value where the deviation followed a normal distribution with mean 0 and standard deviation of 0.02 . This value was chosen to be large enough such that local adaptation, speciation, and ultimately the adaptive radiations progressed within reasonable time (in terms of computational time) but were small enough so that distinct clusters (species) could be detected. After reproduc- 
tion, the parent generation was discarded, and all offspring were born into the habitat of their respective parent but dispersed with probability $d$ to one of the neighboring habitats.

The adaptive radiation in our model saturated at some equilibrium number of species, depending on parameter values. Saturation does not mean that speciation ceases altogether, just that further speciation events are cancelled out by extinctions, caused by demographic stochasticity. We stopped our simulations when the metacommunity was saturated, and we refer to the extant species at this point as the end community and analyzed patterns in overlap between end community sister species. More specifically, the stop criterion was based on the total number of individuals in the system, which is closely linked to total richness (fig. A5; figs. A1-A5 available online). If the mean number of individuals in two sliding windows $(5,000$ time steps long, positioned side by side at the end of the simulation) differed by $<100$ individuals, the simulation was stopped. In other words, we monitored the adaptive radiations during the buildup of diversity, and the length of simulations varied throughout parameter space. All simulations were replicated 99 times. The source code is available at http://dx.doi.org/10.5281/zenodo.18852.

\section{Species Definition and Data Analysis}

The microevolutionary processes (inheritance, mutation, and selection) generated a clustered distribution of individuals in trait space. Clusters moved through trait space following the current selection gradient and occasionally split into new clusters as a result of disruptive selection (Pontarp et al. 2012b). Similar to Pontarp et al. (2012b), we used the clusters as a basis for our species definition. Species identity was treated as a heritable trait, which did not change as long as all individuals within a species had a continuous trait distribution with gaps $<0.1$ (irrespective of in which habitat the individuals occurred). As soon as a gap of at least 0.1 was recorded within a species, that species was split into two by altering the species identity of all individuals. The individuals on one side of the gap were assigned to one unique species number, and all individuals on the other side were assigned to another species number. At this time, the spatial overlap of the newly formed species was recorded (see below). Species gaps were evaluated in this way every 50 generations. The heritability of species identity meant that a species, once identified as such, was never merged with other species, even though clusters sometimes converged in trait space because of convergent trait evolution.

The cluster limit of 0.1 was chosen primarily to agree with what we could easily identify by eye as separate clusters, but there are also biological reasons to why 0.1 - or something close to that value - was suitable. First of all, 0.1 corresponds to five times the standard deviation of the mutations. It is thus highly unlikely that a single mutation can lead to a new species, but a smaller limit would make speciation too easy. Second, a larger gap size than our chosen value, say at 0.2 , means that many speciations that are easily identified by eye are not detected by our algorithm. The smallest niche width $\left(\sigma_{\alpha}\right)$ we used in our simulations was exactly 0.1 . We thus require a separation of not more than one niche width to count a cluster as a species. We also tested the robustness of our results to this cluster limit (see "Robustness Tests" in the appendix).

We calculated the degree of geographical overlap between any two species ( $a$ and $b$ ) as

$$
\frac{\sum_{i} n_{a, i} n_{b, i}}{\sqrt{\sum_{i} n_{a, i}^{2} \sum_{i} n_{b, i}^{2}}}
$$

where $n_{a, i}$ and $n_{b, i}$ are the number of individuals of cluster $a$ and $b$ in habitat $i$, respectively. The sums were taken over all habitats $(i=1 \ldots 5)$. This metric is analogous to the classic niche overlap index (MacArthur and Levins 1967): it is 0 if there is no geographical overlap of the two new species (corresponding to allopatry) and 1 if they have identical geographical distributions of densities (corresponding to perfect sympatry with no geographical segregation). A possible interpretation, which relates this measure to other measures, is that each habitat represents a whole suite of small habitat patches of similar type. The density in each model habitat is then a mean-field approximation of a more fine-grained spatial structure. Species occurrence on the smaller scale is a highly stochastic process with rapid turnover, governed by demographic stochasticity rather than deterministic interactions. The probability of finding two species co-occurring in a small-scale patch is then roughly proportional to the product of their mean-field densities, and the expression in equation (4) would amount to the total proportion of small-scale habitats where two species co-occur.

Finally, we measured the geographical range size of a species as the standard deviation in geographical position of the individuals, where habitats $1-5$ have geographical positions $1-5$, respectively. A species that occurs in only one habitat will, according to this definition, have a geographical standard deviation of 0 , and a species that is distributed uniformly in all five habitats will have a standard deviation of $2^{1 / 2}$. We rescaled the range size such that these extreme values fall between 1 and 5 . With this rescaling, our range size measure can be roughly interpreted as the number of habitats in which a species occurs with more than just a small proportion. To obtain a single value of the range size of a single speciation event, we used the mean range size of the two newly formed species. 


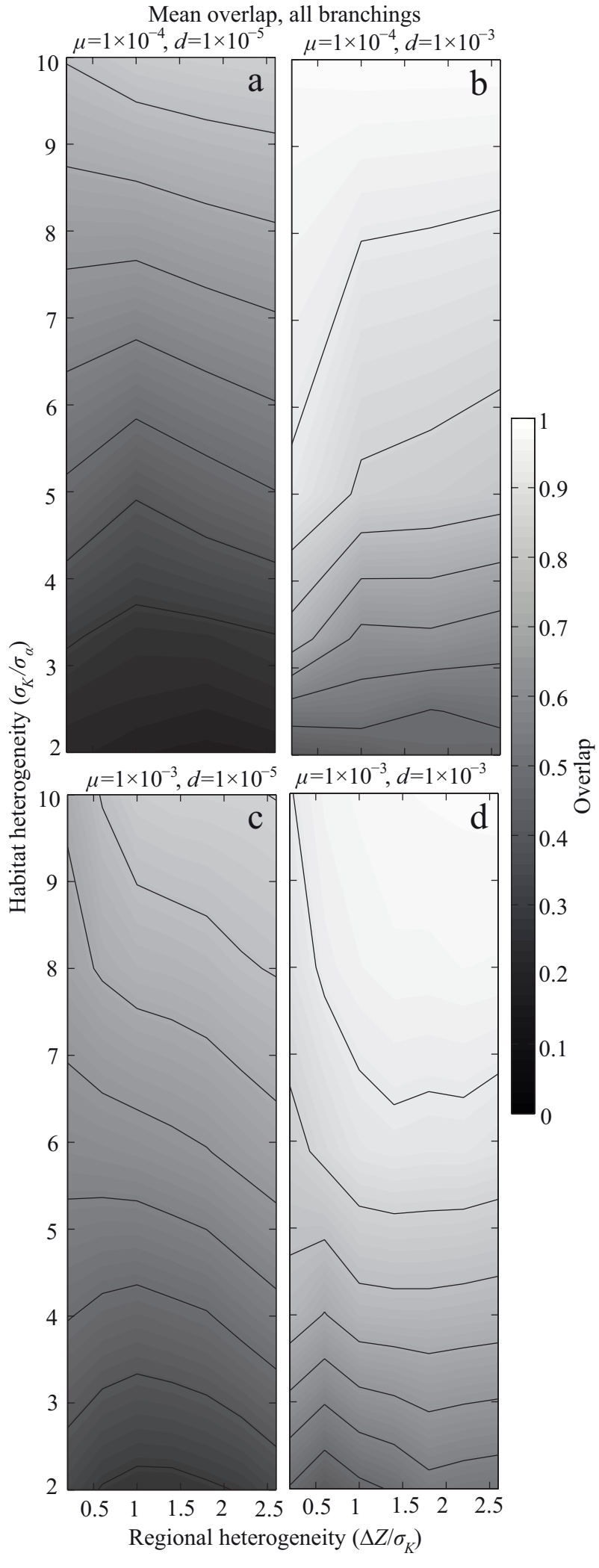

\section{Results}

\section{Biogeographic Patterns of Speciation}

Depending on parameter settings, the mean geographic overlap at speciation covered almost the entire range from no overlap (0) to complete overlap (1). Note, however, that the distribution of overlaps sometimes was highly bimodal and that the change of the mean could be due to a shift in the balance between the two extremes (see below). Figure 2 shows the mean overlap of all speciation events that occurred throughout a radiation as a function of the regional ( $X$-axis) and local ( $Y$-axis) heterogeneity. The four panels show different combinations of high and low dispersal rate $(d)$ and high and low mutation rate $(\mu)$. As expected, the overall level of spatial overlap was higher in the high dispersal cases (fig. $2 b, 2 d$ compared with fig. $2 a$, $2 c$ ). The rate of mutation had a more complicated effect, increasing the overlap when habitat heterogeneity was low and regional heterogeneity was high and decreasing when habitat heterogeneity was high and regional heterogeneity was low (fig. $2 a, 2 b$ compared with fig. $2 c, 2 d$ ). This pattern will be explained below. The mean geographic overlap at speciation increased most clearly with increasing local heterogeneity (fig. 2) because of an enhanced local disruptive selection and increased possibilities for local coexistence (see also Geritz et al. 1998; Dieckmann and Doebeli 1999).

Regional heterogeneity had a somewhat more complicated effect on the geographical overlap. At low levels of local heterogeneity, the geographical separation of newly formed species was maximized when regional heterogeneity was intermediate (lower part of parameter space; fig. 2). An increased regional heterogeneity increases the total number of speciations as a result of a larger number of unique niches available. These new speciations will at intermediate levels of regional heterogeneity be predominately allopatric because of contrasting local selection gradients. When the regional heterogeneity is large, however, species are less adapted to neighboring habitats. Successful dispersal events become less frequent, especially if dispersal is rare, and local sympatric speciation may occur before a successful invader becomes established. This effect is most pronounced if the first colonizer canbecause of strong local competition at low levels of local heterogeneity - monopolize the habitat and thereby prevent further invasions (De Meester et al. 2002; Urban and De Meester 2009). Overall, these results are consistent with

Figure 2: Mean spatial overlap between newly formed species for different dispersal rates $d=10^{-5}(a, c)$ and $d=10^{-3}(b, d)$ and different mutation rates $\mu=10^{-4}(a, b)$ and $\mu=10^{-3}(c, d)$ throughout parameter space. The $X$-axis indicates regional heterogeneity (the difference is resource distributions among habitats, $\left.\Delta Z / \sigma_{K}\right)$, and the $Y$-axis indicates local heterogeneity (potential niche availability, $\sigma_{K} / \sigma_{\alpha}$ ). 
those of Doebeli and Dieckmann (2003), who concluded that the conditions for parapatric speciation, with a small spatial overlap, are most found at intermediate levels of spatial heterogeneity.

At high levels of local heterogeneity, the local disruptive selection is always relatively strong, making sympatric branching more likely throughout the range of regional heterogeneities (fig. 2). When the regional heterogeneity at the same time is low, there is little difference between habitats and two separating branches can readily co-occur over a suite of habitats, which means the range size at speciation is large (fig. A1). As regional heterogeneity increases, the spatial distribution of the two new species becomes less similar, which we record as a lower degree of overlap (fig. 2b). This decreasing trend is reversed if the mutation rate is high (fig. $2 c, 2 d$ ), which enhances local sympatric branching, which in turn has opposing effects at different ends of the regional heterogeneity scale. At the lower end, local branching may occur in a single habitat of a wellspread species, which counts as an overlap below 1. At a higher level of regional heterogeneity, most species are present in only one habitat, and a local branching event counts as fully sympatric. The decreasing trend in overlap is also reversed if the rate of dispersal is decreased (fig. 2a) and for similar reasons. A low rate of dispersal prevents the establishment of new morphs in all suitable habitats, which decreases the recorded overlap of speciations, especially at low regional heterogeneity.

Above, we presented the mean geographic overlap throughout the adaptive radiation. There was, however, a large variation among the speciation events for all parameter settings, except when the mean was close to 0 or 1. The distributions of geographical overlap of new species were sometimes strongly bimodal, as mentioned above (fig. 3). In such cases, a change in the mean overlap corresponds to a shifting balance between the two extremespredominantly sympatric speciation on the one hand and allopatric speciation on the other. The bimodality occurred mainly in parameter settings with large regional heterogeneity and low dispersal. Such circumstances hamper successful establishment in other habitats, especially if it is already occupied by an incipient sister species. Mizera and Meszéna (2003) obtained similar results from a model of evolutionary branching along a continuous spatial gradient. In their model, a decreased spatial tolerance (equivalent to an increased spatial heterogeneity in our model) resulted in species with less spatial overlap (Mizera and Meszena 2003, their fig. 8). An interpretation of these results is that sharp environmental contrasts - or, equivalently, strong trade-offs in habitat-related traits - promotes a dichotomy in biogeographic speciation patterns. The biogeographic mode of speciation is either allopatric or sympatric, in the latter case driven by local disruptive selec- tion. The more precise environmental setting of a particular system, such as the potential for local niche partitioning, will determine which pattern dominates - sympatry or allopatry.

The geographical overlap of speciation was also dependent on when in the cladogenesis they happened. In figure 4, the mean overlap is plotted as a function of the number of species in the system for nine different parameter combinations. When regional heterogeneity was low, there was a clear trend of decreasing overlap at speciation as diversification progressed. This is at least partly due to the increasing local competition, which prevents the establishment of new morphs in all suitable habitats, as discussed above. Another mechanism is the decreasing population size toward the end of a radiation, due to the increased local competition, which makes dispersal and establishment in neighboring habitats less likely. When regional heterogeneity was high, we saw an oscillating mean overlap, matching the temporal patterns of colonization into novel habitats, as the diversity increases (fig. 4; see also fig. 1). A plausible explanation for these patterns is the sequential colonization of habitats, each followed by fast local radiation. The sympatric speciation events that follow colonization will, however, slow down as diversity builds up locally because of the mechanisms mentioned above, leading to these oscillations.

Finally, the range overlap of newly formed species had a tendency to increase with time since speciation, in particular when dispersal was high and local heterogeneity was low (fig. 5). Low local heterogeneity implies in our model strong local competition between similar species and that later ecological divergence promotes coexistence. At low levels of dispersal and high mutation rates, there were many allopatric speciation events simply because spatially separated populations drifted apart (not shown). These species never separated ecologically to a degree that local coexistence was possible. Instead, they could persist in allopatry for long times, without an increase in overlap.

\section{The Geographical Distribution of Sister Species in the Fully Diversified Community}

We next evaluate the possibility to infer the geographical mode of speciation from the range overlap of end community sister species. The linear relationship (computed on data from multiple simulations) between sister species overlap and time since speciation ranged from positive to negative throughout our parameter space, with a tendency for positive trends when regional heterogeneity was low (fig. 6). When analyzing the change in overlap from the time of speciation event until the end of the simulations for specific sister pairs, information that is normally not available in data from natural systems, we also found both 


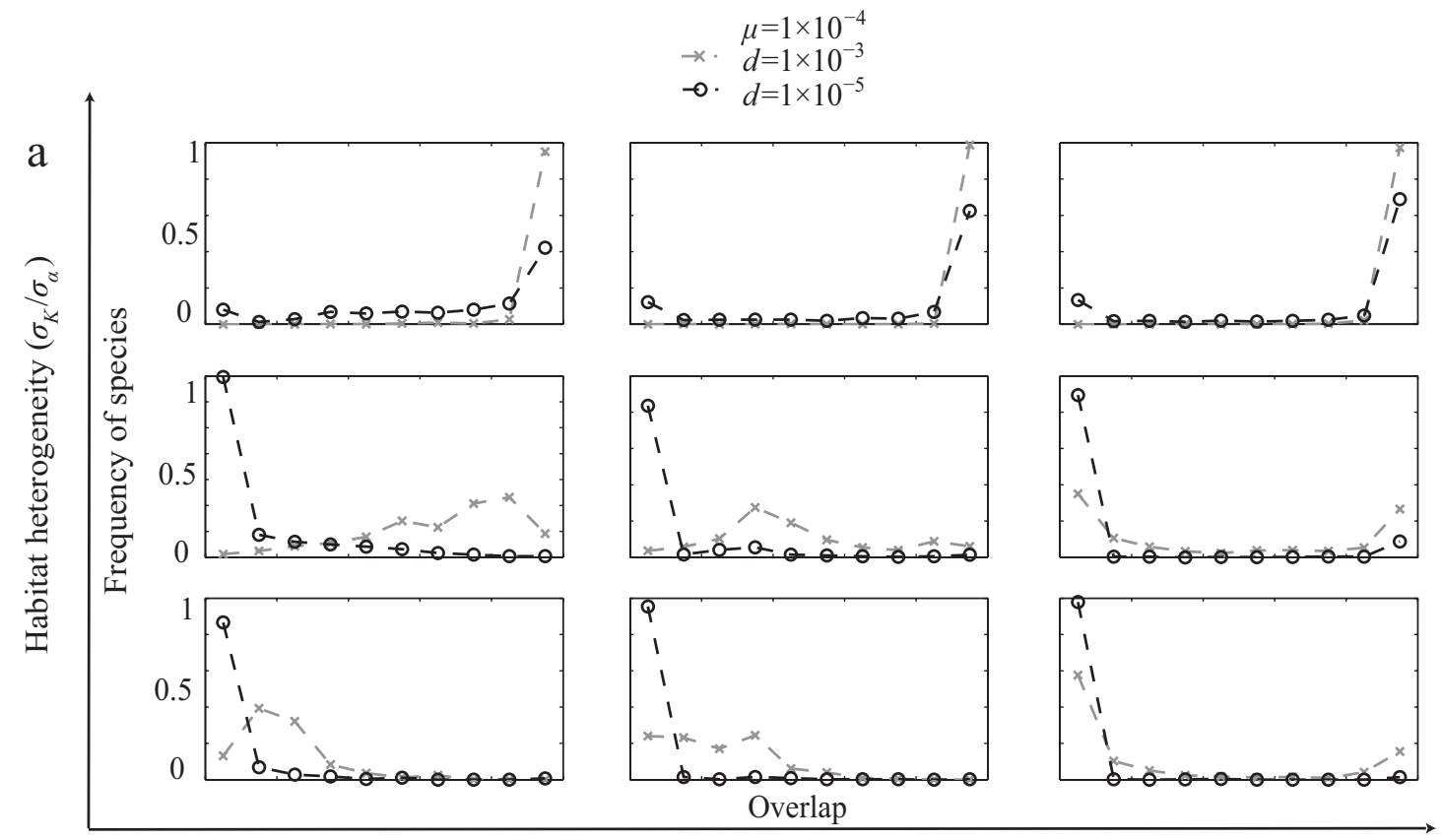

Regional heterogeneity $\left(\Delta Z / \sigma_{K}\right)$

$$
\begin{aligned}
& \mu=1 \times 10^{-3} \\
& * \quad d=1 \times 10^{-3} \\
& \text {-。. } d=1 \times 10^{-5}
\end{aligned}
$$
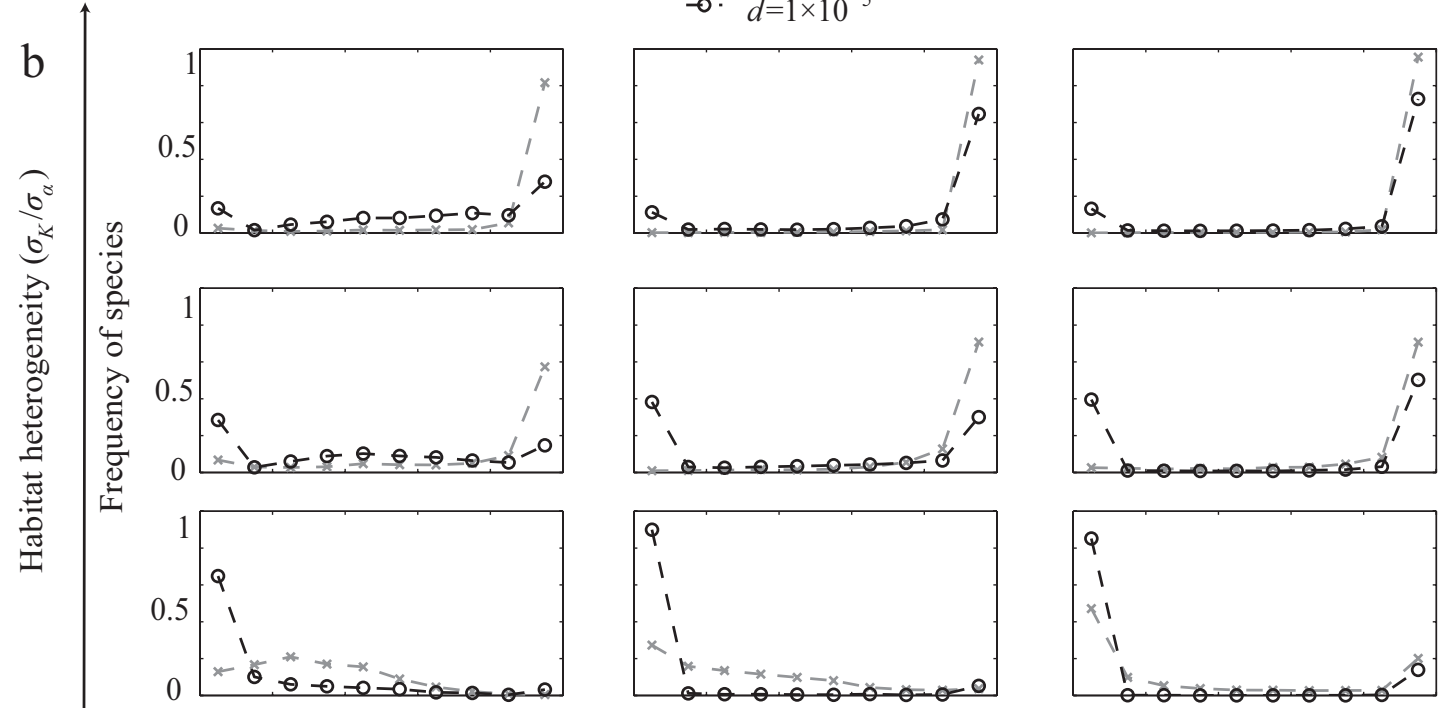

Overlap

Regional heterogeneity $\left(\Delta Z / \sigma_{K}\right)$

Figure 3: Distribution of the geographical overlaps (ranging from 0 to 1 ) at speciation for nine parameter combinations of local and regional habitat heterogeneity. $a$ and $b$ show results from simulations with low $\left(\mu=10^{-4}\right)$ and high $\left(\mu=10^{-3}\right)$ mutation rates. The high dispersal case $\left(d=10^{-3}\right)$ is denoted by gray lines and markers $x$. The low dispersal case $\left(d=10^{-5}\right)$ is denoted by black lines and markers o. Rows (major $Y$-axis) present results for low $\left(\sigma_{K} / \sigma_{\alpha}=2\right)$, intermediate $\left(\sigma_{K} / \sigma_{\alpha}=6\right)$, and high $\left(\sigma_{K} / \sigma_{\alpha}=10\right)$ local heterogeneity. Columns (major $X$-axis) correspond to low $\left(\Delta Z / \sigma_{K}=0.2\right)$, intermediate $\left(\Delta Z / \sigma_{K}=1.4\right)$, and high $\left(\Delta Z / \sigma_{K}=2.6\right)$ regional heterogeneity. 


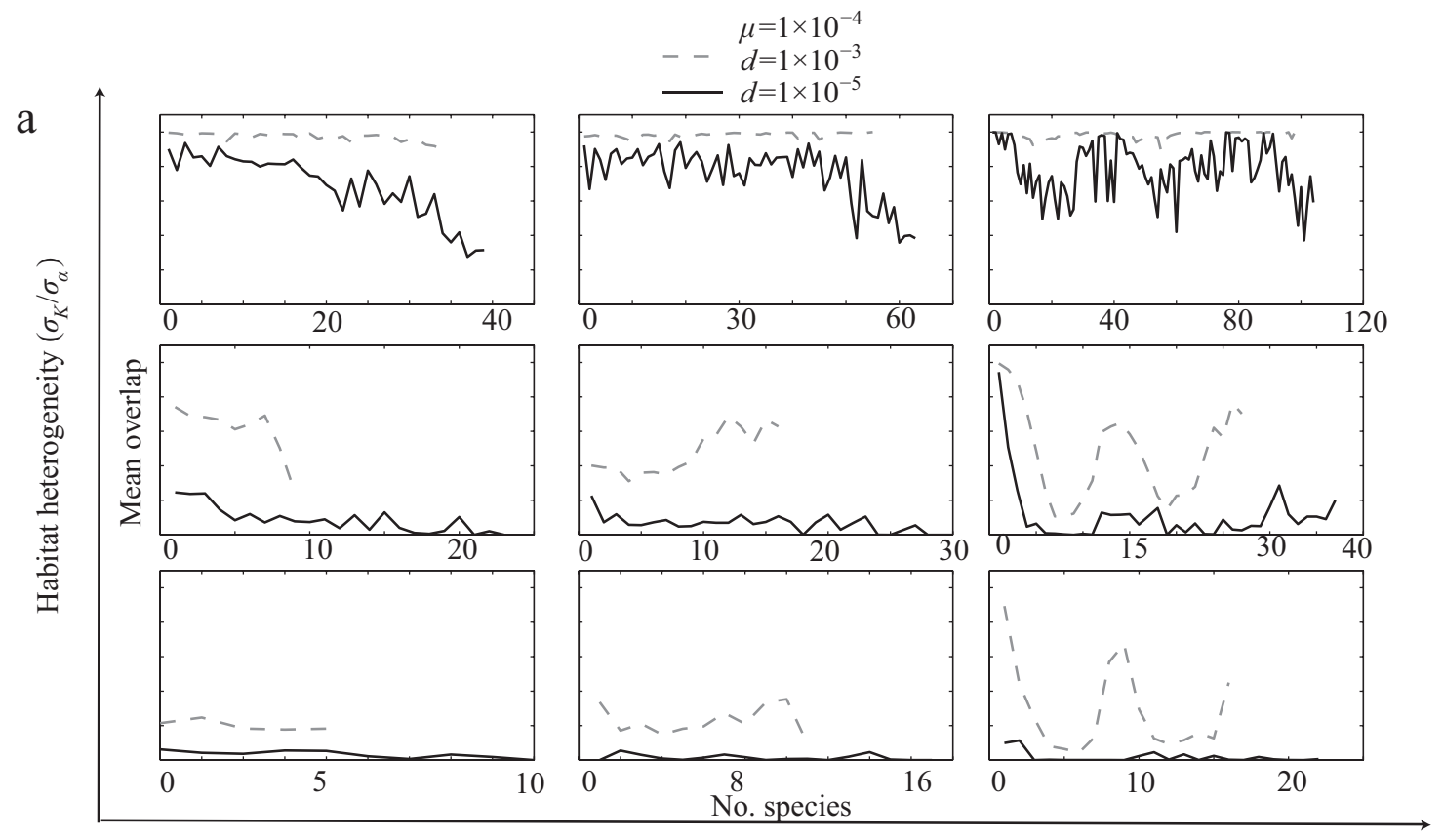

Regional heterogeneity $\left(\Delta Z / \sigma_{K}\right)$

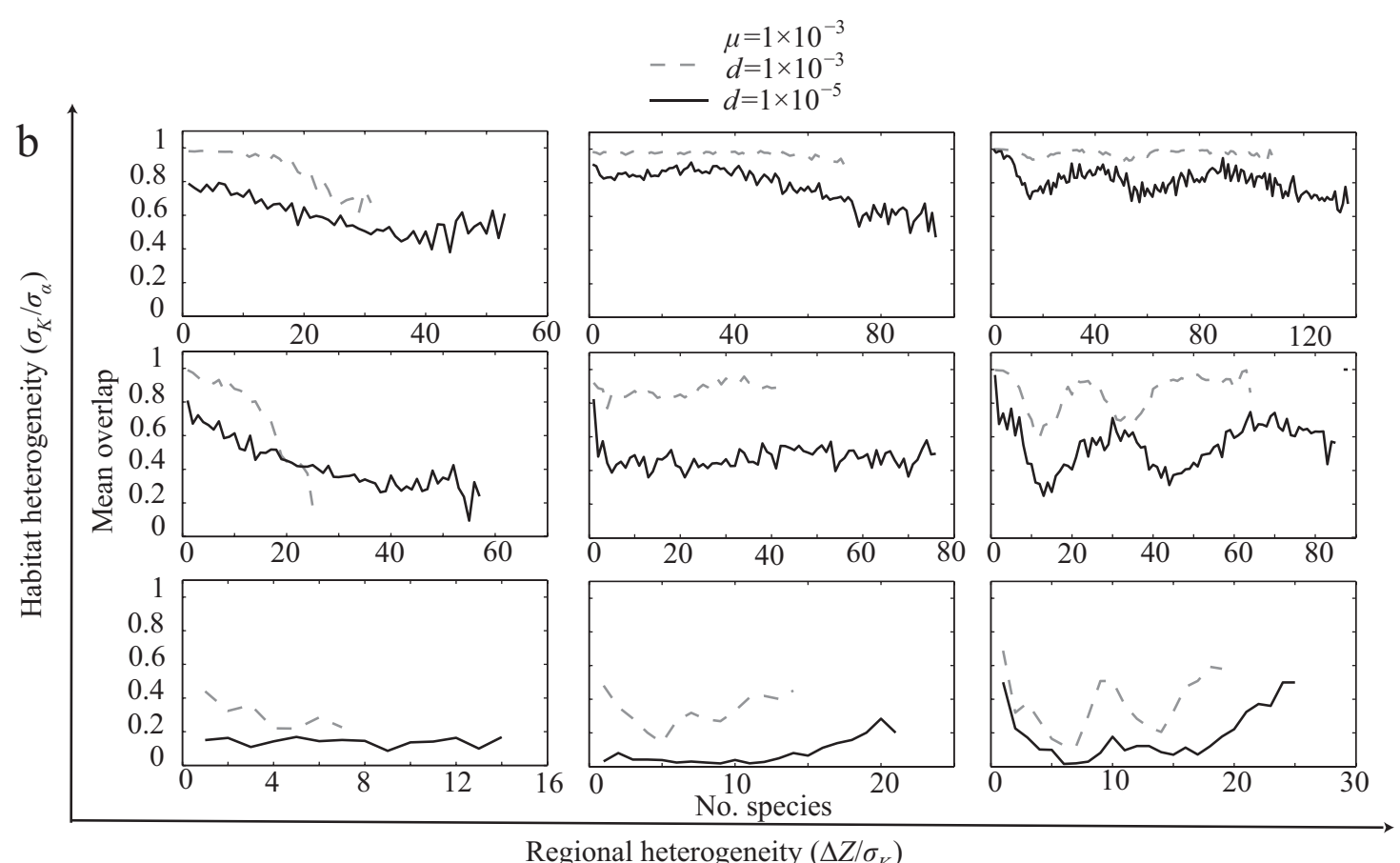

Figure 4: Mean spatial overlap between newly formed species as a function of community saturation (defined as species diversity in the community). $a$ and $b$ show results from simulations with low $\left(\mu=10^{-4}\right)$ and high $\left(\mu=10^{-3}\right)$ mutation rate. The high dispersal case $\left(d=10^{-3}\right)$ is denoted by gray dashed lines. The low dispersal case $\left(d=10^{-5}\right)$ is denoted by black solid lines. Rows (major $Y$-axis) present results for low $\left(\sigma_{K} / \sigma_{\alpha}=2\right)$, intermediate $\left(\sigma_{K} / \sigma_{\alpha}=6\right)$, and high $\left(\sigma_{K} / \sigma_{\alpha}=10\right)$ local heterogeneity. Columns (major $X$-axis) present results for low $\left(\Delta Z / \sigma_{K}=0.2\right)$, intermediate $\left(\Delta Z / \sigma_{K}=1.4\right)$, and high $\left(\Delta Z / \sigma_{K}=2.6\right)$ regional heterogeneity. Note that the species diversity varies in parameter space. 


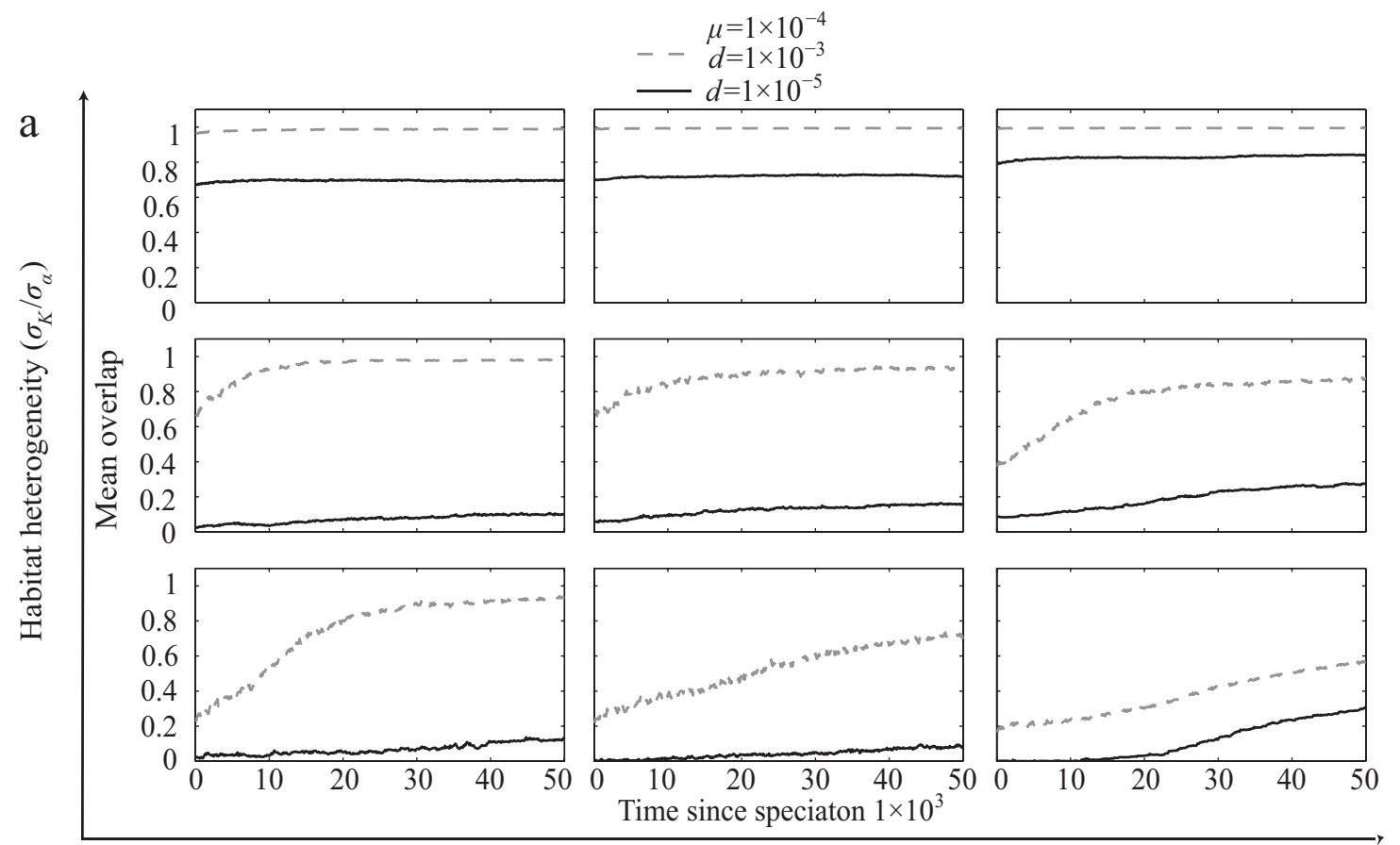

Regional heterogeneity $\left(\Delta Z / \sigma_{K}\right)$

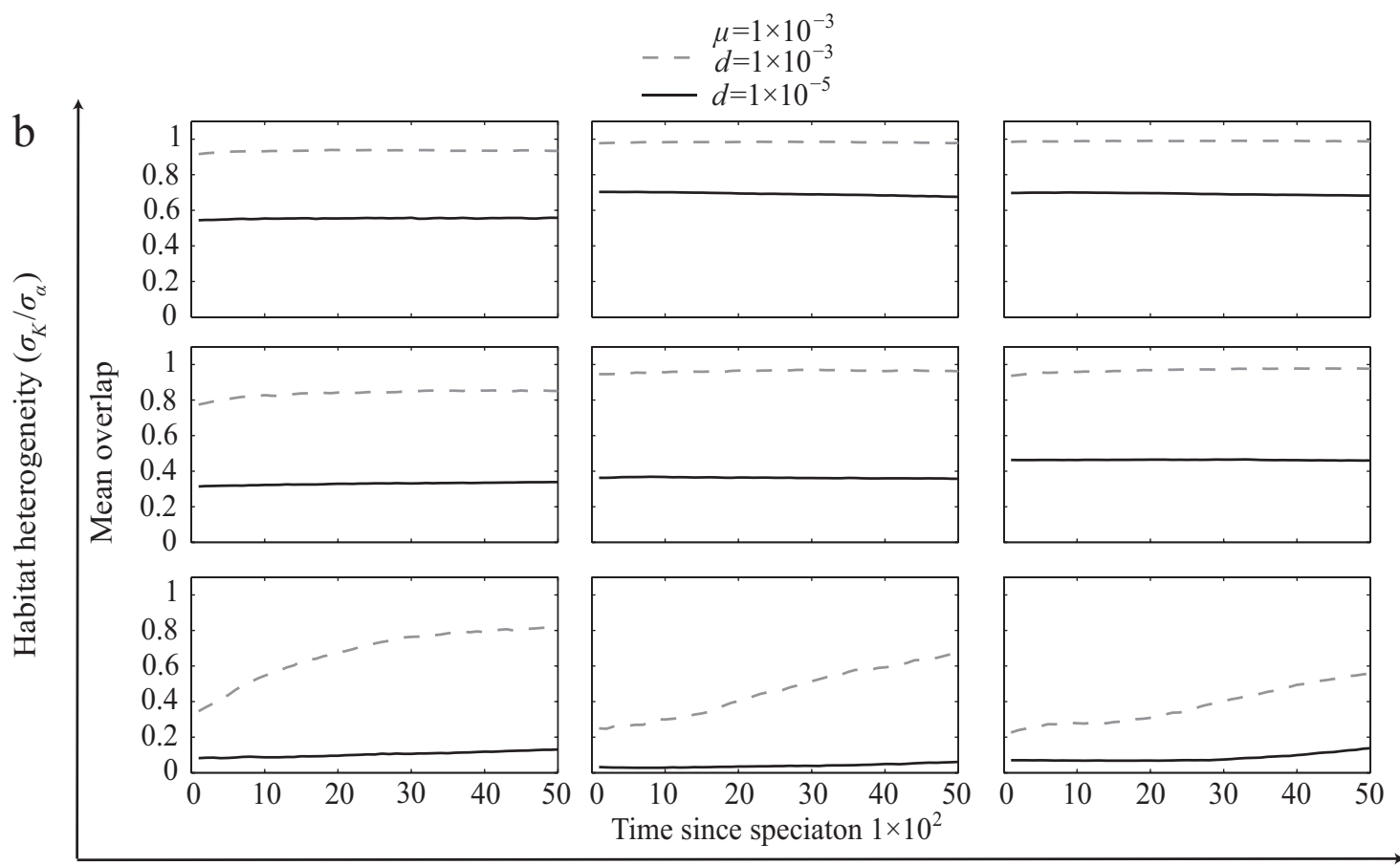

Regional heterogeneity $\left(\Delta Z / \sigma_{K}\right)$

Figure 5: Mean overlap as a function of time since speciation. Only sister species that did not branch again for at least 5,000 time steps were included in the analysis. $a$ and $b$ show results from simulations with low $\left(\mu=10^{-4}\right)$ and high $\left(\mu=10^{-3}\right)$ mutation rate. The high dispersal case $\left(d=10^{-3}\right)$ is denoted by gray dashed lines. The low dispersal case $\left(d=10^{-5}\right)$ is denoted by black solid lines. Rows (major $Y$-axis) present results for low $\left(\sigma_{K} / \sigma_{\alpha}=2\right)$, intermediate $\left(\sigma_{K} / \sigma_{\alpha}=6\right)$, and high $\left(\sigma_{K} / \sigma_{\alpha}=10\right)$ local heterogeneity. Columns (major $X$-axis) present results for low $\left(\Delta Z / \sigma_{K}=0.2\right)$, intermediate $\left(\Delta Z / \sigma_{K}=1.4\right)$, and high $\left(\Delta Z / \sigma_{K}=2.6\right)$ regional heterogeneity. 


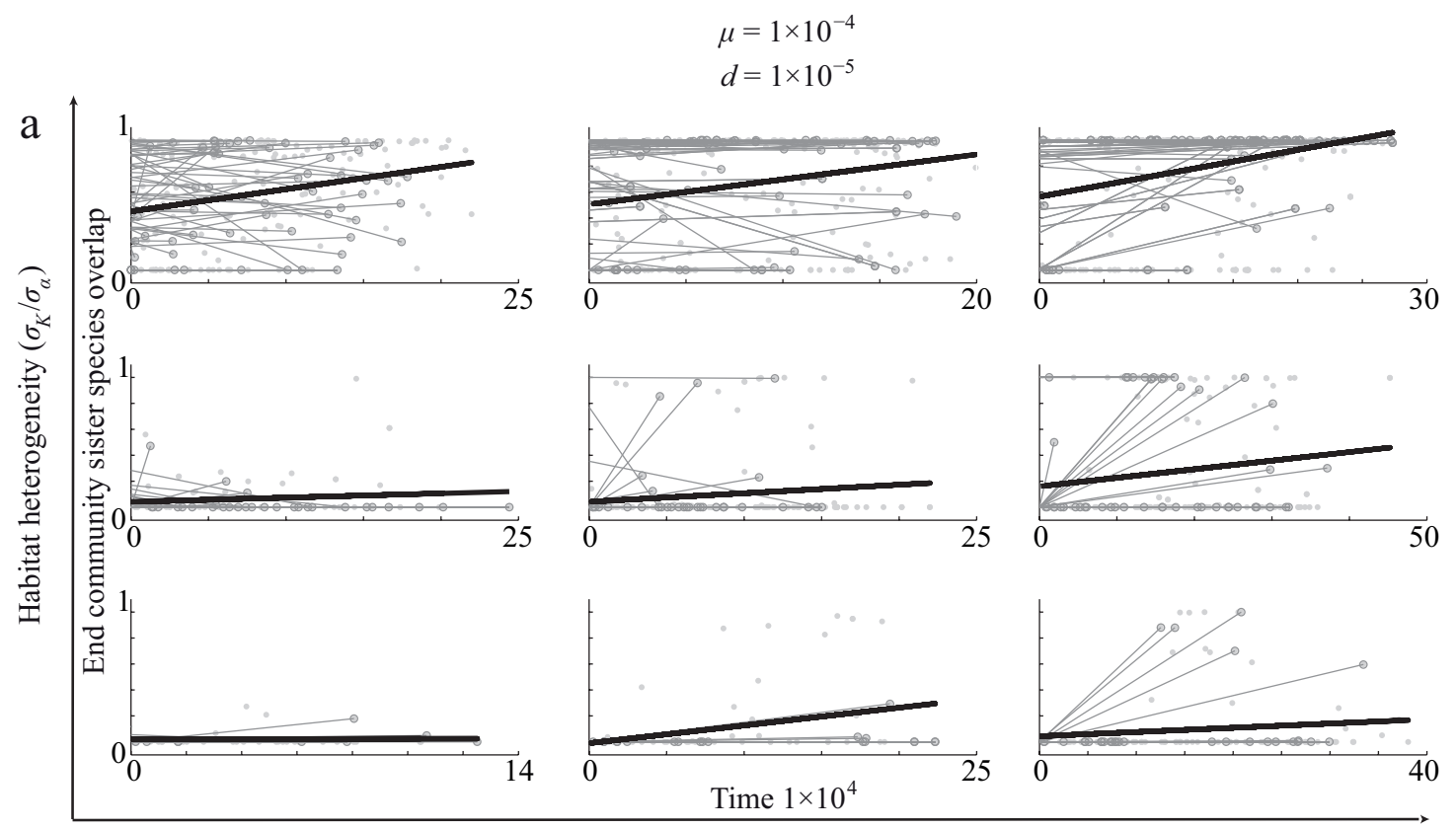

Regional heterogeneity $\left(\Delta Z / \sigma_{K}\right)$

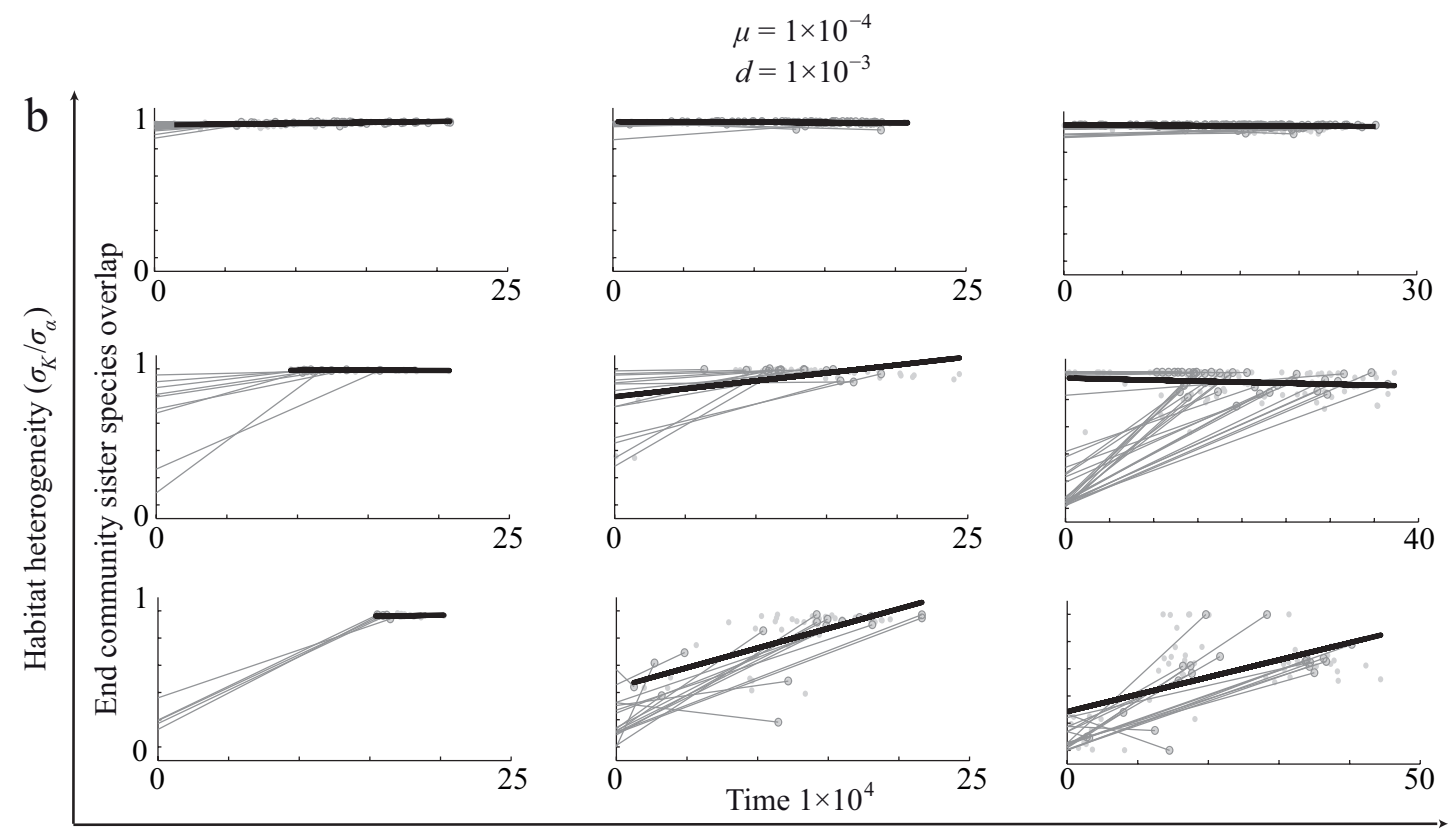

Regional heterogeneity $\left(\Delta Z / \sigma_{K}\right)$

Figure 6: End community sister species overlap as a function of time since speciation in the low mutation rate $\left(\mu=10^{-4}\right)$ case for low dispersal $\left(d=10^{-5} ; a\right)$ and high dispersal $\left(d=10^{-3} ; b\right)$. Data from 20 simulation replicates (light gray dots) with corresponding regression line (black line). The gray lines and circles show end community sister species overlap at speciation (gray line crossing $Y$-axis) versus overlap in the end community (gray circle) for a subset of the data (five replicates). Rows (major $Y$-axis) present results for low $\left(\sigma_{K} / \sigma_{\alpha}=2\right)$, intermediate $\left(\sigma_{K} / \sigma_{\alpha}=6\right)$, and high $\left(\sigma_{K} / \sigma_{\alpha}=10\right)$ local heterogeneity. Columns (major $X$-axis) present results for low $\left(\Delta Z / \sigma_{K}=0.2\right)$, intermediate $\left(\Delta Z / \sigma_{K}=1.4\right)$, and high $\left(\Delta Z / \sigma_{K}=2.6\right)$ regional heterogeneity. 
positive and negative trends (gray lines in fig. 6). More specifically, figure $6 a$ shows settings with a low rate of dispersal and a low rate of mutation. Postspeciation range shifts are in this case rare and highly stochastic. If they occur, they can both increase and decrease the range overlap (fig. $6 a$ ). Figure $6 b$ shows the case with a low mutation rate but a relatively high dispersal rate. This is the case with the strongest relationships, which may lead to correct inference on speciation biogeography. Especially the cases with a low local heterogeneity (fig. $6 b$, bottom row) show strong positive trends, correctly indicating a low geographic overlap at speciation. These are also the cases when we generally found long-term, persistent trends in range overlap with time since speciation (fig. 5). At higher levels of local heterogeneity, the postspeciation range shifts were too rapid to be detected on a long timescale (fig. $6 b$, middle and top rows). The end community sister species were in those cases all sympatric, indicating sympatric speciation. That conclusion is, however, misleading at intermediate levels of local heterogeneity, where the overlap at speciation was on average less than sympatric (fig. $6 b$, middle row; see also fig. $2 b)$. At the higher mutation rate $\left(\mu=10^{-4}\right)$, the patterns were similar but slightly more noisy (appendix, fig. A3).

A technical comment: the power of any statistical test heavily depends on the sample size. In our case, the circumstances that lead to consistent trends are exactly the cases with very few sister species in the end communities. A regression test with data from a single adaptive radiation is therefore futile. We simply have to conjecture that a larger-scale system, real or simulated, would produce patterns consistent with our results but with larger statistical power.

\section{Discussion}

\section{The Model Captures the Essentials}

Simulating the assembly of evolving communities and metacommunities is a powerful tool when trying to understand the link between process and pattern in natural systems (Gotelli et al. 2009). For example, several studies have investigated the drivers of richness patterns between regions (Goldberg et al. 2005; Roy and Goldberg 2007; Yoder and Nuismer 2010). Others have studied community assembly along environmental gradients (McPeek 2008; Stegen et al. 2009, 2012a, 2012b), and Birand et al. (2012) examined speciation, extinction, and range sizes. Similar to these studies, we simulate assembly processes such as competition and coevolution of multiple species (see also Nuismer et al. 2010) and analyze the emergent biogeographical patterns. We combined a trait-based eco-evolutionary modeling approach, similar to previous studies on speciation in single-species systems (Dieckmann and Doebeli 1999), and large-scale metacommunity modeling (e.g., McPeek 2007; Stegen and Hurlbert 2011; Pontarp et al. 2012b). The theoretical foundations of this approach are well established (Dieckmann et al. 2004; Vincent and Brown 2005; Gavrilets 2014): density-dependent competition for available resources and regional spatial structure determine the rate of adaptation, local radiation, and patterns of local and global coexistence. The simulation approach also allows for stochastic demographic effects, especially when population sizes are small and the fitness landscape is shallow (Johansson and Ripa 2006; Claessen et al. 2007). This means that the most relevant ecological and evolutionary processes - such as dispersal, selection, local adaptation, speciation, and demographic stochasticity, identified by Vellend (2010) — are included in our model.

\section{Model Assumptions}

Our model is built from basic principles of eco-evolutionary dynamics, an approach that is well established and considered useful when studying general rules and patterns (Gavrilets 2014). However, this generality is associated with assumptions that may or may not impact the results. First, similar to, for example, Haller et al. (2013), we neglect genetic mechanisms and sexual reproduction. Our results consequently do not encompass mechanisms such as recombination (which may prevent speciation) and nonecological speciation (e.g., Dobzhansky-Mueller incompatibilities). Simulation studies show that asexual speciation is generally easier than the speciation of sexually reproducing species in both sympatry (Dieckmann and Doebeli 1999) and parapatry (Doebeli and Dieckmann 2003; Heinz et al. 2009). The evolution of mating traits leading to speciation beyond a first speciation event is, however, poorly understood. As an example, if a magic trait mechanism evolves such that there is strong assortment on the evolving ecological trait, further speciation is prevented because of strong stabilizing sexual selection (Ripa 2009). Bolnick (2006) circumvents this problem by letting all necessary genetic variation be available in the ancestral population. This leads to a rapid segregation into a suite of coexisting species but is nevertheless followed by evolutionary stasis. Aguilee et al. (2009) present a model where an adaptive radiation proceeds despite a strong assortative mating. This is made possible through strong external forcing - populations go through periods of complete allopatry and complete sympatry. Sympatric speciation is in principle impossible in this model. Birand et al. (2012) study a model where speciation is driven by local adaptation coupled with the evolution of habitat preference as well as mating traits. Recognizable species evolve, but reproductive isolation is almost entirely accomplished through strong habitat preferences, not mate prefer- 
ence (Birand et al. 2012). Notably, sympatric speciation is not possible since local coexistence is not possible in this model. Rettelbach et al. (2013) demonstrate that several biogeographic speciation modes indeed are possible within a single model, even including the complications of sexual reproduction, but their results still only apply to a single speciation event. We could, within the limited current understanding, think of no simple model design that would with high probability generate the type of scenarios we were after. Because our goal was to study the fundamental ecoevolutionary drivers of the geographical mode of speciation during adaptive radiations in heterogeneous habitats, we thus used an asexual model, focusing on the ecological drivers of speciation. The effects of sexual reproduction and different mating systems will have to be left for future studies.

Second, the linear arrangement of habitats and the stepping stone dispersal algorithm do not apply universally. Both altitudinal and latitudinal gradients do, however, involve a linear arrangement of habitats, where the end habitat cannot be colonized without colonizing intermediate habitats first. Furthermore, there is a range of other realistic scenarios that our model does not consider (e.g., overlapping generations, temporally variable environments, and trophic interactions). These are all relevant and important to consider when we aim to understand the full scope of the speciation process. However, trying to include more detail in our model is not practical and would detract from our goal of understanding the fundamental eco-evolutionary drivers of geographical mode of speciation during adaptive radiations in heterogeneous landscapes.

\section{Local Heterogeneity Enhances Local Branching}

The relationship between local heterogeneity and sympatric speciation is well understood in theory (Geritz et al. 1998; Dieckmann and Doebeli 1999). In our model, this process is contingent on the quotient $\sigma_{K} / \sigma_{\alpha}$, which we vary in our analysis. There is also empirical support for the conclusion that local habitat heterogeneity enhances local speciation rate (Hobohm 2000; Roos et al. 2004; Hughes and Eastwood 2006). The mechanistic explanation for such patterns has mainly been that a large local heterogeneity corresponds to low local competition between diverging types, which enhances local coexistence and ultimately sympatric speciation. In other words, speciation is a local process driven by character displacement caused by local competition. The resultant species will be sympatric and, at least initially, endemic to one habitat.

We also recognize that our fixed parameters, $K_{0}$ and $r$, may affect local diversification. $K_{0}$ regulates the number of individuals in each species. The number of species in a local community is saturated when the speciation rate equals the extinction rate. A small $K_{0}$ leads to a higher extinction rate because of demographic stochastic events and a slower speciation rate for the same reason (Claessen et al. 2007). A higher $K_{0}$ will thus lead to a larger number of species at saturation. A high $r$ value increases the strength of selection, which may increase the speciation rate and therefore species richness in the saturated community. Despite these effects, however, we do not expect that changing $K_{0}$ or $r$ will affect the qualitative patterns in our results.

\section{Regional Heterogeneity Has Ambiguous Effects}

Increased regional heterogeneity has two different effects. First, it facilitates allopatric speciation, which is theoretically well understood in the sense that an increased difference between habitats implies stronger disruptive selection and enhanced conditions for evolutionary branching, at least up to a point where the difference between habitats is too large (Meszéna et al. 1997; Nilsson and Ripa 2010). Second, larger differences between habitats promote habitat monopolization and local, sympatric speciation (DeMeester 1996). Large differences also cause direct habitat filtering (Pontarp et al. 2012b). As the habitats become increasingly distinct, it becomes increasingly difficult to invade neighboring habitats, and local niches are predominantly filled through local radiation after colonization. Many local communities appear to be habitat filtered (Emerson and Gillespie 2008; Vamosi et al. 2009; Pontarp et al. 2012a). For example, high-altitude habitats are occupied by sets of closely related species among bees (Hoiss et al. 2012), hummingbirds (Graham et al. 2009), and ants (Machac et al. 2011).

\section{Dispersal Enhances Spatial Overlap and Decreases the Number of Species}

An increased dispersal rate resulted in increased range overlap at speciation (fig. 2) as well as increased range sizes at speciation. Apart from that, the dispersal rate only marginally influenced the effects of the other parameters in the model. A number of studies using a similar approach have shown that the possibility for evolutionary branching decreases with increased rate of dispersal (e.g., Brown and Pavlovic 1992; Mizera and Meszena 2003; Parvinen and Egas 2004). Figure 4 indicates that increased dispersal does decrease the number of species in the fully saturated community. Few previous studies have addressed the effect of dispersal on the spatial overlap at speciation (but see, e.g., Pigot et al. 2010). Others have shown that gene flow, caused by dispersal, can disrupt speciation (Slatkin 1973; Felsenstein $1981 b$; Lenormand 2002). The reason for this can, for example, be genetic recombination and loss of reproductive isola- 
tion. Here we do not take such mechanisms into account, since we use an asexual model.

\section{Speciation Mode Is Not an Inherent Species Trait}

Our simulations show that allopatric, sympatric, and intermediate types of speciations can occur at the same time, within a single adaptive radiation. Small shifts in local circumstances, such as the invasion or local extinction of a competitor, can shift the speciation pattern from one to the other. We also found consistent temporal trends in overlap at speciation, also explained by the changed ecological and evolutionary circumstance throughout a radiation. Thus, it cannot be expected that any particular clade or organism type always displays the same biogeographic pattern at speciation.

\section{The Distribution of Geographic Range Overlap Is Sometimes Bimodal}

For some parameter settings, the distribution of geographic overlap at speciation was bimodal, such that the overlap was either very small (allopatric) or very large (sympatric). The tendency for bimodality was the strongest when regional heterogeneity was high and dispersal was low. In line with previous studies (e.g., Mizera and Meszéna 2003; Rettelbach et al. 2013), these results suggest that sharp environmental contrasts and strong trade-offs in habitat-related traits promote a dichotomy in biogeographic speciation patterns, while less sharp contrasts may facilitate a more continuous distribution of speciation mode ranging from sympatry to allopatry. While this conclusion is rather intuitive, the exact details of when and how to expect a bimodality of range overlaps remains an open question. We have demonstrated the phenomenon in a large-scale simulation, and others have made ground-breaking numerical work on smaller systems (Mizera and Meszéna 2003; Rettelbach et al. 2013), but a more complete understanding will have to be left for future studies, let alone the ultimate confrontation with data.

\section{The Overlap of Extant Sister Species Says Little about the Overlap at Speciation}

Patterns of the spatial distribution of sister species and measures of time since speciation have been used to infer the biogeographic speciation pattern (allo- or sympatric). A positive relationship between sister species overlap and time since speciation is commonly interpreted as allopatric speciation followed by increased overlap due to range shifts (Losos and Glor 2003). A negative relationship has been interpreted as sympatric speciation followed by decreased overlap, also due to range shifts (Barraclough and
Vogler 2000; Quenouille et al. 2011). However, these interpretations are controversial and depend on assumptions of, for example, limited or constant range shifts in species and constant eco-evolutionary circumstances throughout the cladogenesis. Losos and Glor (2003) argued that the observed large variation in geographical overlap is due to postspeciation evolution and repeated, more or less random range shifts. Contrasting patterns with both positive and negative trends in, for example, African rain forest trees (Couvreur et al. 2011) and studies where no pattern is found (Nakazato et al. 2010) make the big picture ambiguous.

We did see positive trends in overlap as a function of time since speciation, especially when the dispersal rate was high, the local heterogeneity was low, and the mutation rate was low (fig. $6 b$ ). In other cases, however, the relationship was often too noisy to be informative, or the range shifts were too fast to be detected on a long timescale (as argued by Losos and Glor [2003]). Furthermore, the end community sister species may not be representative of all the speciation events throughout an adaptive radiation. In our model, they tended to arise in the late part of the adaptive radiation (fig. A2), and there were in some cases trends in mean overlap over time (fig. 4). Given the highly idealized situation-no observation error, a single evolving trait without complexities such as genetic constraints or environmental fluctuations - we conclude that the slope of the relationship between time since speciation and current range overlap is sufficiently variable to discourage any strong inference on the biogeography at speciation.

\section{Conclusions}

The link between the past mode of speciation and current biogeography of sister species is of fundamental interest to evolutionary biologists. The understanding of the causal link between environmental properties, eco-evolutionary processes, and biogeography do, for example, underpin current methods that use the geographic distribution of extant species to infer evolutionary processes. The very fundamentals of this link are, however, largely unknown (e.g., Losos and Glor 2003; Fitzpatrick and Turelli 2006), and in line with previous criticism, our results discourage such inference. We show that overlap at speciation can vary continuously with local and regional environmental heterogeneity, dispersal, and species richness. The mean overlap may also vary during the course of a single radiation, and the speciation events that give rise to end community sister species are thus a biased sample of all speciation events throughout the radiation. This provides explanations for why it can be difficult to infer, for example, the geographical speciation mode from patterns in ex- 
tant species biogeography. With this said, we do, however, see the value of inferring processes that are difficult to study in nature from observational data, given that such inference is well founded in theory. We thus encourage both empirical and theoretical studies to explore the link between process and pattern during assumptions and scenarios different from the ones studied here.

\section{Acknowledgments}

This study was financially supported by grants from the Swedish Research Council (to J.R., P.L., and M.P.) and the Royal Physiographic Society (to M.P.). The simulations were performed on resources provided by the Swedish National Infrastructure for Computing at the Center for Scientific and Technical Computing at Lund University. We also thank L. Harmon, J. Rosindell, and anonymous reviewers for comments and suggestions that improved the initial version of this article.

\section{Literature Cited}

Aguilee, R., D. Claessen, and A. Lambert. 2009. Allele fixation in a dynamic metapopulation: founder effects vs refuge effects. Theoretical Population Biology 76:105-117.

Barraclough, T. G., and A. P. Vogler. 2000. Detecting the geographical pattern of speciation from species-level phylogenies. American Naturalist 155:419-434.

Birand, A., A. Vose, and S. Gavrilets. 2012. Patterns of species ranges, speciation, and extinction. American Naturalist 179:1-21.

Bolnick, D. I. 2006. Multi-species outcomes in a common model of sympatric speciation. Journal of Theoretical Biology 241:734744.

Brown, J. S., and N. B. Pavlovic. 1992. Evolution in heterogeneous environments: effects of migration on habitat specialization. Evolutionary Ecology 6:360-382.

Brown, J. S., and T. L. Vincent. 1987. A theory for the evolutionary game. Theoretical Population Biology 31:140-166.

Christiansen, F. B., and V. Loeschcke. 1980. Evolution and intraspecific exploitative competition. 1. One-locus theory for small additive gene effects. Theoretical Population Biology 18:297-313.

Claessen, D., J. Andersson, L. Persson, and A. M. de Roos. 2007. Delayed evolutionary branching in small populations. Evolutionary Ecology Research 9:51-69.

Couvreur, T. L. P., H. Porter-Morgan, J. J. Wieringa, and L. W. Chatrou. 2011. Little ecological divergence associated with speciation in two African rain forest tree genera. BMC Evolutionary Biology 11:296.

Coyne, J. A., and H. A. Orr. 2004. Speciation. Sinauer, Sunderland, MA.

Day, T. 2000. Competition and the effect of spatial resource heterogeneity on evolutionary diversification. American Naturalist 155: 790-803.

De Meester, L. 1996. Local genetic differentiation and adaptation in freshwater zooplankton populations: patterns and processes. Ecoscience 3:385-399.
De Meester, L., A. Gomez, B. Okamura, and K. Schwenk. 2002. The monopolization hypothesis and the dispersal-gene flow paradox in aquatic organisms. Acta Oecologica 23:121-135.

Dieckmann, U., and M. Doebeli. 1999. On the origin of species by sympatric speciation. Nature 400:354-357.

Dieckmann, U., M. Doebeli, J. A. J. Metz, and D. Tautz. 2004. Adaptive speciation. Cambridge University Press, Cambridge.

Doebeli, M., and U. Dieckmann. 2003. Speciation along environmental gradients. Nature 421:259-264.

Eaton, M. D., J. Soberon, and T. Peterson. 2008. Phylogenetic perspective on ecological niche evolution in American blackbirds (family Icteridae). Biological Journal of the Linnean Society 94: 869-878.

Elith, J., and J. R. Leathwick. 2009. Species distribution models: ecological explanation and prediction across space and time. Annual Review of Ecology, Evolution, and Systematics 40:677-697.

Emerson, B. C., and R. G. Gillespie. 2008. Phylogenetic analysis of community assembly and structure over space and time. Trends in Ecology and Evolution 23:619-630.

Felsenstein, J. 1981a. Continuous-genotype models and assortative mating. Theoretical Population Biology 19:341-357.

- 1981b. Skepticism towards Santa Rosalia, or why are there so few kinds of animals. Evolution 35:124-138.

Fitzpatrick, B. M., and M. Turelli. 2006. The geography of mammalian speciation: mixed signals from phylogenies and range maps. Evolution 60:601-615.

Fry, J. D. 2003. Multilocus models of sympatric speciation: Bush versus Rice versus Felsenstein. Evolution 57:1735-1746.

Gavrilets, S. 2014. Models of speciation: where are we now? Journal of Heredity 105:743-755.

Gavrilets, S., and A. Vose. 2005. Dynamic patterns of adaptive radiation. Proceedings of the National Academy of Sciences of the USA 102:18040-18045.

Geritz, S. A. H., E. Kisdi, G. Meszena, and J. A. J. Metz. 1998. Evolutionarily singular strategies and the adaptive growth and branching of the evolutionary tree. Evolutionary Ecology 12:35-57.

Goldberg, E. E., K. Roy, R. Lande, and D. Jablonski. 2005. Diversity, endemism, and age distributions in macroevolutionary sources and sinks. American Naturalist 165:623-633.

Gotelli, N. J., M. J. Anderson, H. T. Arita, A. Chao, R. K. Colwell, S. R. Connolly, D. J. Currie, et al. 2009. Patterns and causes of species richness: a general simulation model for macroecology. Ecology Letters 12:873-886.

Graham, C. H., J. L. Parra, C. Rahbek, and J. A. McGuire. 2009. Phylogenetic structure in tropical hummingbird communities. Proceedings of the National Academy of Sciences of the USA 106: 19673-19678.

Haller, B. C., R. Mazzucco, and U. Dieckmann. 2013. Evolutionary branching in complex landscapes. American Naturalist 182:E127E141.

Heinz, S. K., R. Mazzucco, and U. Dieckmann. 2009. Speciation and the evolution of dispersal along environmental gradients. Evolutionary Ecology 23:53-70.

Hobohm, C. 2000. Plant species diversity and endemism on islands and archipelagos, with special reference to the Macaronesian Islands. Flora 195:9-24.

Hoiss, B., J. Krauss, S. G. Potts, S. Roberts, and I. Steffan-Dewenter. 2012. Altitude acts as an environmental filter on phylogenetic composition, traits and diversity in bee communities. Proceedings of the Royal Society B: Biological Sciences 279:4447-4456. 
Hughes, C., and R. Eastwood. 2006. Island radiation on a continental scale: exceptional rates of plant diversification after uplift of the Andes. Proceedings of the National Academy of Sciences of the USA 103:10334-10339.

Johansson, J., and J. Ripa. 2006. Will sympatric speciation fail due to stochastic competitive exclusion? American Naturalist 168:572-578

Kisdi, E. 2002. Dispersal: risk spreading versus local adaptation. American Naturalist 159:579-596.

Krug, P. J. 2011. Patterns of speciation in marine gastropods: a review of the phylogenetic evidence for localized radiations in the sea. American Malacological Bulletin 29:169-186.

Leibold, M. A., M. Holyoak, N. Mouquet, P. Amarasekare, J. M. Chase, M. F. Hoopes, R. D. Holt, et al. 2004. The metacommunity concept: a framework for multi-scale community ecology. Ecology Letters 7:601-613.

Leibold, M. A., and J. Norberg. 2004. Biodiversity in metacommunities: plankton as complex adaptive systems? Limnology and Oceanography 49:1278-1289.

Lenormand, T. 2002. Gene flow and the limits to natural selection. Trends in Ecology and Evolution 17:183-189.

Losos, J. B., and R. E. Glor. 2003. Phylogenetic comparative methods and the geography of speciation. Trends in Ecology and Evolution 18:220-227.

Losos, J. B., and D. Schluter. 2000. Analysis of an evolutionary speciesarea relationship. Nature 408:847-850.

MacArthur, R. H., and R. Levins. 1967. Limiting similarity convergence and divergence of coexisting species. American Naturalist 101:377-385.

Machac, A., M. Janda, R. R. Dunn, and N. J. Sanders. 2011. Elevational gradients in phylogenetic structure of ant communities reveal the interplay of biotic and abiotic constraints on diversity. Ecography 34:364-371.

Mayr, E. 1942. Systematics and the origin of species. Cambridge University Press, Cambridge.

McPeek, M. A. 2007. The macroevolutionary consequences of ecological differences among species. Palaeontology 50:111-129.

- 2008. The ecological dynamics of clade diversification and community assembly. American Naturalist 172:E270-E284.

Meszéna, G., I. Czibula, and S. Geritz. 1997. Adaptive dynamics in a 2-patch environment: a toy model for allopatric and parapatric speciation. Journal of Biological Systems 5:265-284.

Mizera, F., and G. Meszena. 2003. Spatial niche packing, character displacement and adaptive speciation along an environmenta gradient. Evolutionary Ecology Research 5:363-382.

Nakazato, T., D. L. Warren, and L. C. Moyle. 2010. Ecological and geographic modes of species divergence in wild tomatoes. American Journal of Botany 97:680-693.

Nilsson, J., and J. Ripa. 2010. Adaptive branching in source-sink habitats. Evolutionary Ecology 24:479-489.

Nosil, P., D. J. Funk, and D. Ortiz-Barrientos. 2009. Divergent selection and heterogeneous genomic divergence. Molecular Ecology 18:375-402.

Nuismer, S. L., R. Gomulkiewicz, and B. J. Ridenhour. 2010. When is correlation coevolution? American Naturalist 175:525-537.

Ogden, R., and R. S. Thorpe. 2002. Molecular evidence for ecological speciation in tropical habitats. Proceedings of the National Academy of Sciences of the USA 99:13612-13615.

Parvinen, K., and M. Egas. 2004. Dispersal and the evolution of specialisation in a two-habitat type metapopulation. Theoretical Population Biology 66:233-248
Peterson, A. T., J. Soberon, and V. Sanchez-Cordero. 1999. Conservatism of ecological niches in evolutionary time. Science 285:12651267.

Phillimore, A. B., C. D. L. Orme, G. H. Thomas, T. M. Blackburn, P. M. Bennett, K. J. Gaston, and I. P. F. Owens. 2008. Sympatric speciation in birds is rare: insights from range data and simulations. American Naturalist 171:646-657.

Pigot, A. L., A. B. Phillimore, I. P. F. Owens, and C. D. L. Orme 2010. The shape and temporal dynamics of phylogenetic trees arising from geographic speciation. Systematic Biology 59:660673.

Pontarp, M., B. Canback, A. Tunlid, and P. Lundberg. 2012a. Phylogenetic analysis suggests that habitat filtering is structuring marine bacterial communities across the globe. Microbial Ecology 64:8-17.

Pontarp, M., J. Ripa, and P. Lundberg. 2012b. On the origin of phylogenetic structure in competitive metacommunities. Evolutionary Ecology Research 14:269-284

Quenouille, B., N. Hubert, E. Bermingham, and S. Planes. 2011. Speciation in tropical seas: allopatry followed by range change. Molecular Phylogenetics and Evolution 58:546-552.

Rettelbach, A., M. Kopp, U. Dieckmann, and J. Hermisson. 2013. Three modes of adaptive speciation in spatially structured populations. American Naturalist 182:E215-E234.

Ripa, J. 2009. When is sympatric speciation truly adaptive? an analysis of the joint evolution of resource utilization and assortative mating. Evolutionary Ecology 23:31-52.

Rocha, L. A., D. R. Robertson, J. Roman, and B. W. Bowen. 2005 Ecological speciation in tropical reef fishes. Proceedings of the Royal Society B: Biological Sciences 272:573-579.

Roos, M. C., P. J. A. Kessler, S. R. Gradstein, and P. Baas. 2004. Species diversity and endemism of five major Malesian islands: diversityarea relationships. Journal of Biogeography 31:1893-1908.

Roy, K., and E. E. Goldberg. 2007. Origination, extinction, and dispersal: integrative models for understanding present-day diversity gradients. American Naturalist 170(suppl.):S71-S85.

Slatkin, M. 1973. Gene flow and selection in a cline. Genetics 75 733-756.

Stegen, J. C., B. J. Enquist, and R. Ferriere. 2009. Advancing the metabolic theory of biodiversity. Ecology Letters 12:1001-1015

. 2012a. Eco-evolutionary community dynamics: covariation between diversity and invasibility across temperature gradients. American Naturalist 180:E110-E126.

Stegen, J. C., R. Ferriere, and B. J. Enquist. 2012b. Evolving ecological networks and the emergence of biodiversity patterns across temperature gradients. Proceedings of the Royal Society B: Biological Sciences 279:1051-1060.

Stegen, J. C., and A. H. Hurlbert. 2011. Inferring ecological processes from taxonomic, phylogenetic and functional trait $\beta$-diversity. PLoS ONE 6:e20906.

Thompson, J. N. 2005. The geographic mosaic of coevolution. University of Chicago Press, Chicago.

Urban, M. C. 2004. Disturbance heterogeneity determines freshwater metacommunity structure. Ecology 85:2971-2978.

Urban, M. C., and L. De Meester. 2009. Community monopolization: local adaptation enhances priority effects in an evolving metacommunity. Proceedings of the Royal Society B: Biological Sciences 276:4129-4138.

Urban, M. C., M. A. Leibold, P. Amarasekare, L. De Meester, R. Gomulkiewicz, M. E. Hochberg, C. A. Klausmeier, et al. 2008. 
The evolutionary ecology of metacommunities. Trends in Ecology and Evolution 23:311-317.

Urban, M. C., and D. K. Skelly. 2006. Evolving metacommunities: toward an evolutionary perspective on metacommunities. Ecology 87:1616-1626.

Vamosi, S. M., S. B. Heard, J. C. Vamosi, and C. O. Webb. 2009. Emerging patterns in the comparative analysis of phylogenetic community structure. Molecular Ecology 18:572-592.

Vellend, M. 2010. Conceptual synthesis in community ecology. Quarterly Review of Biology 85:183-206.
Vincent, T. L., and J. S. Brown. 2005. Evolutionary game theory, natural selection and Darwinian dynamics. Cambridge University Press, New York.

Yoder, J. B., and S. L. Nuismer. 2010. When does coevolution promote diversification? American Naturalist 176:802-817.

Associate Editor: Luke J. Harmon Editor: Judith L. Bronstein

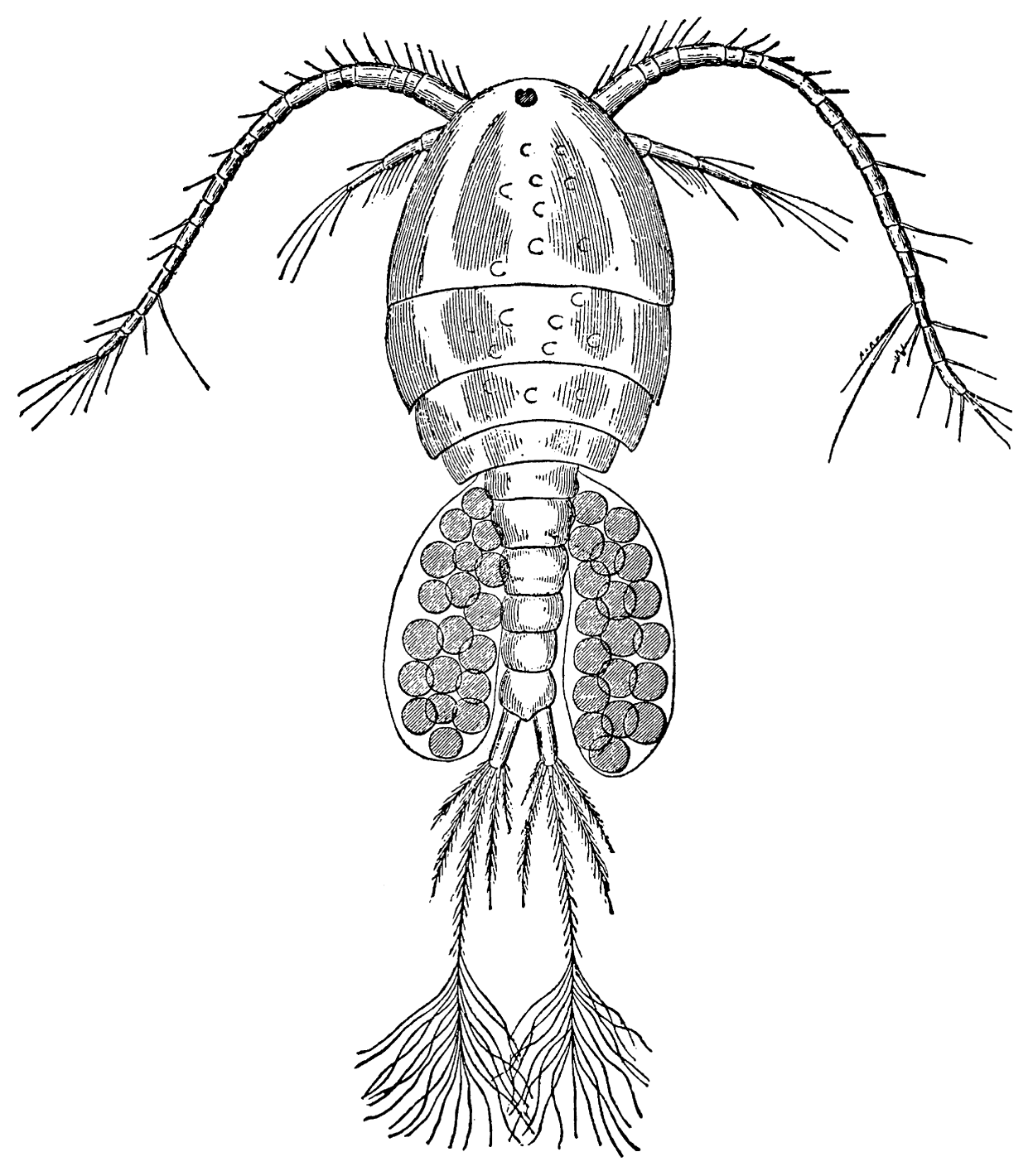

"The collector of fresh-water specimens is constantly meeting unexpected forms, especially among the smaller organisms, and of these no order of animals furnishes a wider variety or more curious adaptations than the fresh-water Crustaceans embraced in the old group Entomostraca... To the microscopist particularly they are available as a never-failing field for study, since a cup of water from almost any source will contain abundant material for a day's work." From "Fresh-Water Entomostraca" by C. L. Herrick (The American Naturalist, $1879,13: 620-628)$. 